\title{
An Obstacle Detection Algorithm Based on Ultrasonic Sensors for Autonomous Land Vehicle
}

\author{
Huihai Cui ${ }^{1}$, Yan $\mathrm{Li}^{1 *}$ and Jinze $\mathrm{Liu}^{2}$ \\ ${ }^{1}$ College of Mechatronics Engineering and Automation, National University of Defense Technology, Changsha, Hunan, China \\ ${ }^{2}$ College of Information Science and Engineering, Central South University, Changsha, Hunan, China \\ ${ }^{*}$ Corresponding author
}

\begin{abstract}
An dynamic filtering based obstacle detection algorithm is proposed for the navigation and control of Autonomous Land Vehicle (ALV). The algorithm detects the obstacles using sequential sonar data from dual sonar sensors. The sonar model is described at first. Then the obstacles' features, depicted as lines, are extracted. Finally a dynamic data filtering algorithm is described, in which the sonar return data is firstly processed through dynamic filtering using the orientation and the trajectory information of the vehicle. The algorithm's validity is approved through a field test in cross-country environment.
\end{abstract}

Keywords-Autonomous Land Vehicle; ultrasonic sensor; obstacle detection; dynamic filtering

\section{INTRODUCTION}

It is a fundamental capability for Autonomous Land Vehicle (ALV) to acquire the environment information and build the local obstacle map through various onboard sensors. Obstacle detection devices, used to be installed on ALV, include: vision, LiDAR, millimeter-wave radar, ultrasonic or infrared sensors. Sonar sensor has many merits compared to other popular range sensors, such as: cheap, accurate range readings, unaffected by light intensity, etc. However, sonar sensor suffers from bad angular resolution, specular reflections, and cross-talk, etc., which limit its application usually in near range obstacle avoidance in indoor environment[1][2]. Some researcher even make the conclusion that sonar sensor is not suitable for cross-country ALV applications.

The ALV navigation and control applications in crosscountry environment require sonar sensor to detect the obstacles with the size of $40 \mathrm{X} 40 \mathrm{~cm}$, distributed in the range of $10 \sim 15 \mathrm{~m}$, and with the acceptable distance error limited in $5 \mathrm{~cm}$. To meet the above requirements, a large-range obstacle detection algorithm using sequential sonar data, is proposed, in which the problem of obstacle feature extraction, false data filtering, and obstacle localization, is processed. Dual sonar sensors are used to measure the distance and the orientation of the obstacle lying in the overlapped aperture angle area. The obstacles' features, depicted as lines, are firstly extracted. Then the features all over the movement path of the ALV, are recognized and localized through a dynamic filtering method, in which the sonar return data is firstly processed through static filtering according to the geometric relationship of the ultrasonic array, then, dynamic filtering is executed using the orientation and the trajectory information of the vehicle.

The paper is organized as: Part II introduces the sonar model and discusses the description of the obstacle feature and the obstacle map; Part III presents the obstacle detection algorithm; and, Part IV gives the experiment result.

\section{OBSTACLE FEATURE EXTRACTION}

\section{A. Sonar Modeling}

According to the velocity of ultrasonic, the smallest cycle time of sonar return is $60 \mathrm{~ms}$ in $10 \mathrm{~m}$ range. Therefore, the traditional obstacle detection mode of ultrasonic sensor, in which only one obstacle detected in one cycle, can hardly meet either the requirement of large range obstacle detection or that of multi-sensor information fusion for ALV in complex crosscountry environment. Fortunately, different from linear laser radar, ultrasonic sensor belongs to planar sensor, which can receive all the sonar returns in the radiation range. According to the requirement of large range obstacle detection, a new type of large range sonar sensor has been developed, which can output all distances and intensities of the sonar returns from multiple obstacles during one detect cycle. A single sonar return $\mathrm{R} 1$, is represented by an arc centered at the position of the sonar sensor a, with radius R1, which is limited in the range of aperture angle , as shown by Figure1a. A group of sonar returns detected in one detect cycle, similarly, are represented by a cluster of concentric arcs, as shown by FIGURE 1

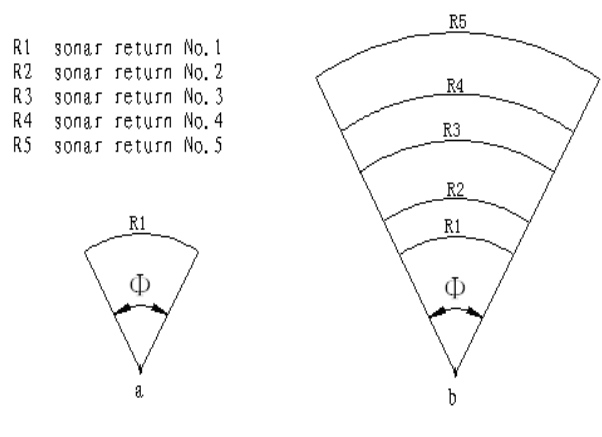

FIGURE I. SONAR MODELING

The amplitude of the sonar signal appears to exponentially attenuate as the following equation:

$$
A(t)=A_{0} \exp \left(-2 \times 10^{-13} \times C \cdot f^{2} \cdot t\right)
$$


Where, $\mathbf{A}$ depicts the sonar amplitude, $\mathrm{A}_{0}$ depicts the sonar amplitude at the probe diffuse surface of the sonar sensor unit, $\mathrm{f}$ depicts the sonar frequency $(\mathrm{MHz}), \mathrm{C}$ depicts the sonar velocity.

\section{B. Obstacle Feature Extraction}

Given that the distance of the obstacle lying in the overlapped aperture area of the two ultrasonic sensors is much larger than the length of the baseline, the geometric features of obstacle are represented by points and lines, satisfy:

1)Dot-like obstacle can be detected only if it lies in the range of the aperture angle of the sonar sensor;

2)Linear obstacle can be detected only if the vertical line from sonar sensor to the object, lies in the range of aperture angle.

Because the angular resolution of the sonar sensor is low, it is difficult to localize the object for single sensor. Therefore, sonar sensor array is needed. Without loss of the generalization, only the dual-sonar array is discussed, and the obstacle detection algorithm consider only those object lie in the overlapped area of the two sonar's aperture angle ranges.

Ellipse localization algorithm is one of the classical ultrasonic localization algorithms. The principle of ellipse localization algorithm is briefly introduced.

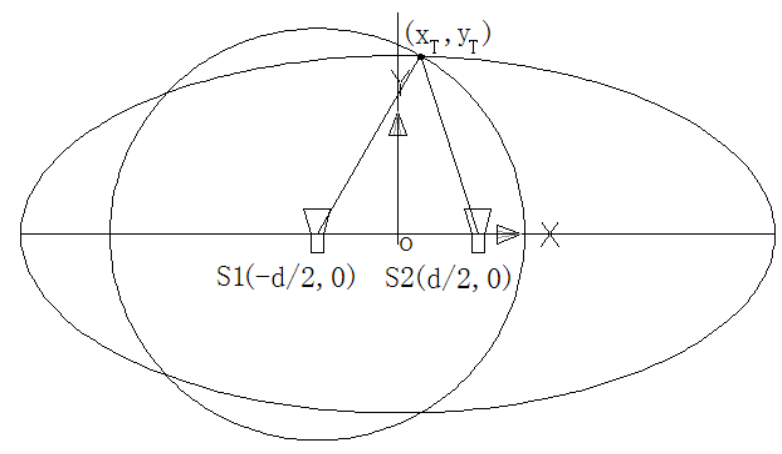

FIGURE II. ELLIPSE LOCALIZATION PRINCIPLE

Given the linear array made up of two identical sonar sensor, respectively named S1、S2. The position coordinates of the S1、S2 are illustrated as FIGURE 2. The position coordinates of the obstacle is supposed as $\left(x_{T}, y_{T}\right)$. Suppose TOF time $t_{i j}(i, j=1,2)$ is the time from the senor $S_{i}$ transmitting a sonar signal to the sensor $S_{j}$ receiving the sonar return, $r_{i j}=v t_{i j}$ is the passing distance of the sonar signal, which is transmitted by $S_{i}$, reflected by obstacle, and received by $S_{j}, v$ is the velocity of sonar wave transmitting. Given an obstacle is located in the far field of the ultrasonic sensors, satisfying the far field constraint of . As for the sensor S1, the coordinates of the obstacle should satisfy the following formula:

$$
x_{T}=\frac{r_{11} r_{12}-r_{12}^{2}}{2 d}, y_{T}=\frac{\sqrt{\left(r_{12}^{2}-d^{2}\right)} \square \sqrt{d^{2}-\left(r_{11}-r_{12}\right)^{2}}}{2 d}
$$

\section{OBSTACle Detection Algorithm}

To get desired obstacle localization effect, it is not enough just improving the performance of the sonar sensor, filtering the measured data is also necessary. In this paper, the sequential sonar return data is processed through the dynamic filtering method using the orientation and the trajectory information of the vehicle.

\section{A. Dynamic Ultrasonic Array}

So called dynamic ultrasonic array is a four unit array dynamically constructed by each group of sonar sensors, using the movement continuity and the known orientation of the vehicle, as shown in FIGURE 3.
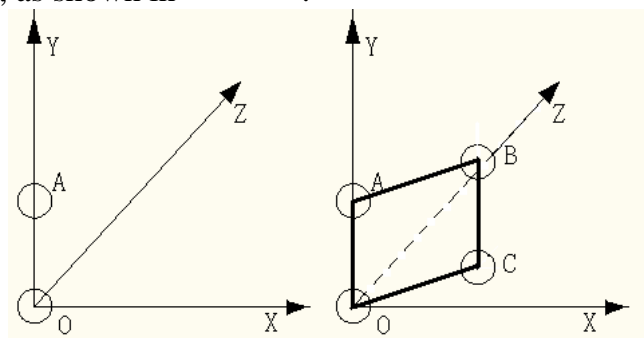

FIGURE III. DYNAMIC SONAR ARRAY: LEFT: A SONAR SENSOR GROUP LAYOUT IN STATIC STATUS; RIGHT: SONAR ARRAY MADE UP OF A GROUP OF SENSOR UNITS

Given the distance a between the sensor $\mathrm{O}$ and the sensor $\mathrm{A}$, the movement distance $\mathrm{b}$ of the vehicle during one detection cycle, then the sensor $\mathrm{A}$ move to $\mathrm{B}$, and the sensor $\mathrm{O}$ move to $\mathrm{C}$. Because the cycle time is very short (counted in $\mathrm{ms}$ ), so distance b is very small. Suppose the height of the vehicle have not been varied, then four points OABC form a rectangular sonar array. The dynamic sonar array is used to measure the location of the obstacle. According to the return time $\left\{t_{1}, t_{2}, t_{3}, t_{4}\right\}$, relating to four sonar sensors, $\{\mathrm{O} 、 \mathrm{~A}$ 、 C、 $\mathrm{B}\}$, and the location of the obstacle, $(x, y, z)$, in the coordinate frame of the sonar array, the localization equations are established as follows:

$$
\left\{\begin{array}{c}
x^{2}+y^{2}+z^{2}=v^{2} t_{1}^{2} \\
x^{2}+(y-a)^{2}+z^{2}=v^{2} t_{2}^{2} \\
(x-b)^{2}+y^{2}+z^{2}=v^{2} t_{3}^{2} \\
(x-a)^{2}+(y-b)^{2}+z^{2}=v^{2} t_{4}^{2}
\end{array}\right.
$$

The location ( $x, y, z)$ of the obstacle, can be solved from above formula.

B. Algorithm Description

The Dynamic Filtering Algorithm:() 
1) Initialize Vehicle Location $\left(X_{0}^{G}\right)$;

2) Acquire the queue of the measurement data: $\left\{T_{1}^{0}, T_{2}^{0}, T_{3}^{0}, T_{4}^{0}\right\}$

3) Static filtering $\left\{T_{1}^{0^{\prime}}, T_{2}^{0^{\prime}}, T_{3}^{0^{\prime}}, T_{4}^{0^{\prime}}\right\}=\operatorname{SFilter}\left(T_{1}^{0}, T_{2}^{0}, T_{3}^{0}, T_{4}^{0}\right)$

4) Acquire the obstacle location estimation: $O_{0}=\operatorname{OLocate}\left(T_{1 i}^{0^{\prime}}, T_{3 j}^{0^{\prime}}\right)$;

5) Transform to the Global Coordinate: $O_{0}^{G}=$ Vehicle $\left(O_{0}, X_{0}^{G}\right)$

6) Build the weight relationship table:

$<O, P>=<O_{0}^{G}, 1>$

7) 7 for $k=1$ to $K$

8) Get Vehicle Location $\left(X_{k}^{G}\right)$;

9) Acquire the queue of the measurement data :

$\left\{T_{1}^{k}, T_{2}^{k}, T_{3}^{k}, T_{4}^{k}\right\}$

10) Static filtering

$\left\{T_{1}^{k^{\prime}}, T_{2}^{k^{\prime}}, T_{3}^{k^{\prime}}, T_{4}^{k^{\prime}}\right\}=\operatorname{SFilter}\left(T_{1}^{k}, T_{2}^{k}, T_{3}^{k}, T_{4}^{k}\right)$

11) Dynamic filtering

$\left\{T_{1}^{k^{\prime}}, T_{2}^{k^{\prime}}\right\}=\operatorname{DFilter}\left(T_{1}^{k^{-1} 1^{\prime}}, T_{2}^{k^{-1} 1^{\prime}}, T_{1}^{k^{\prime}}, T_{2}^{k^{\prime}}, X_{k}^{G}\right)$

$\left\{T_{3}^{k^{\prime}}, T_{4}^{k^{\prime}}\right\}=\operatorname{DFilter}\left(T_{3}^{k-1^{\prime}}, T_{4}^{k-1^{\prime}}, T_{3}^{k^{\prime}}, T_{4}^{k^{\prime}}, X_{k}^{G}\right)$

12) Acquire the obstacle location estimation:

$O_{k}=$ OLocate $\left(T_{1 i}^{k^{\prime}}, T_{3 j}^{k^{\prime}}\right)$;

13) Transform to the Global Coordinate :

$O_{k}^{G}=$ Vehicle $\left(O_{k}, X_{k}^{G}\right)$

14) If $\left(O_{k}^{G}=O_{i}\right) P_{i}=P_{i}+1$

15) if( $\left.\left\|O_{k}^{G}-X_{k}^{G}\right\|<L\right)$

16) Delete $O_{k}^{G}$ from the list

17) end

18) else

19) $<O, P>=<O, P>+<O_{k}^{G}, 1>$

20) end

21) end

22) if $\left(P_{i}>\right.$ W)output $O_{i}^{G} 23$ return

\section{EXPERIMENT RESULT}

To test the algorithm, an obstacle detection test in crosscountry environment was executed, in which the ultrasonic sensors are installed on the ALV platform (错误! 未找到引 用源。). The results show that, in cross-country environment, the algorithm dramatically reduced the specular reflection, cross-talk, and environment noise, at the same time, the distance and direction of the obstacle was measured with high accuracy.

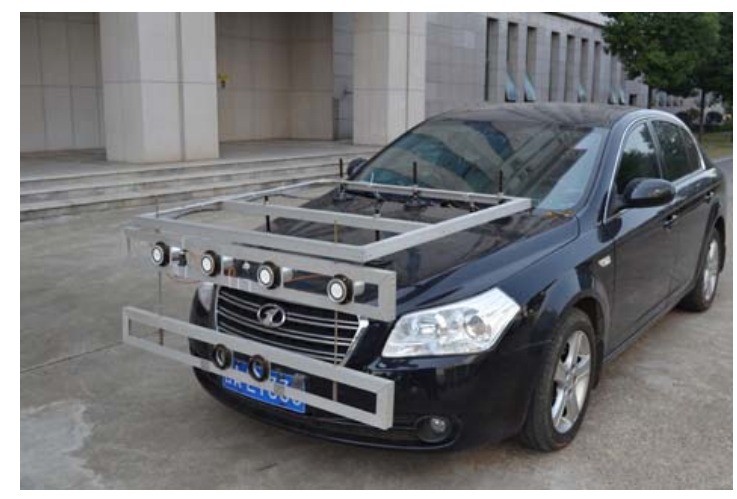

(a) the ultrasonic sensor platform

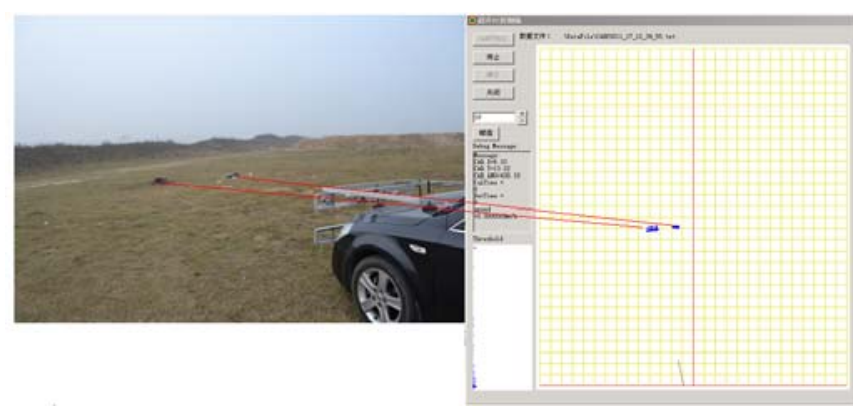

(b). the field obstacle environment and the obstacle map

FIGURE IV. THE OBSTACLE DETECTION EXPERIMENT RESULT

TABLE I. MEASURED DATA AND ERROR

\begin{tabular}{|c|c|c|c|c|c|c|}
\hline $\begin{array}{c}\text { Obstacle } \\
\text { ID }\end{array}$ & $\begin{array}{c}\text { Expected } \\
\text { distance } \\
(\mathrm{m})\end{array}$ & $\begin{array}{c}\text { Expected } \\
\text { angle } \\
\text { range } \\
(\mathrm{deg})\end{array}$ & $\begin{array}{c}\text { Measured } \\
\text { distance } \\
(\mathrm{m})\end{array}$ & $\begin{array}{c}\text { Measured } \\
\text { angle } \\
\text { range } \\
(\mathrm{deg})\end{array}$ & $\begin{array}{c}\text { Distance } \\
\text { error } \\
(\mathrm{cm})\end{array}$ & $\begin{array}{c}\text { Angle } \\
\text { error } \\
(\mathrm{deg})\end{array}$ \\
\hline 1 & 3.65 & 83.72 & 3.64 & 83.94 & 1 & 0.22 \\
\hline 2 & 5.28 & 88.15 & 5.25 & 88.99 & 3 & 0.84 \\
\hline 3 & 8.58 & 93.05 & 8.55 & 92.30 & 3 & 0.75 \\
\hline 4 & 9.20 & 86.15 & 9.16 & 86.83 & 4 & 0.68 \\
\hline 5 & 13.9 & 90.67 & 13.95 & 90.94 & 5 & 0.27 \\
\hline
\end{tabular}

\section{ACKNOWLEDGMENT}

The author wishes to thank the IEEE for providing this template and all colleagues who previously provided technical support.

\section{REFERENCES}

[1] Fodor, M., Liska, O. "Design and realization sensorial system on detection obstacle" SAMI 2010 8th IEEE International Symposium on Applied Machine Intelligence and Informatics , Herl'any, Slovakia, 2010,pp. 243 - 245 
[2] Hongbo Wang, Ke Yu, Bingyi Mao, "Self-localization and Obstacle Avoidance for a Mobile Robot” Neural Computer and Application, 2009 18 pp.495-506

[3] Yu-Cheol Lee, Jong Hwan Lim , Dong-Woo Cho andWan Kyun Chung, “Sonar Map Construction for Autonomous Mobile Robots Using a Data Association Filter” Advanced Robotics No. 23 (2009) 185-201

[4] J. Tardos, J. Neira. "Robust mapping and localization in indoor environments using sonar data[J]. "International Journal of Robotics Research, 2002, 21(4),pp.311 - 330.

[5] Ballard D H. "Generalizing the Hough transform to detect arbitrary shapes”[J]. Pattern Recognition, 1981, 13(2) : 111-122

[6] Califano A, "Mohan R Multidimensional indexing for recognizing visual shape" [J]. IEEE transactions on Pattern Alysis and Machine Intelligence, 1991, 16(4) : 28-34.

[7] Chau C P, Siu W C. “Adaptive dual-point Hough transform for object recognition”[J]. Computer Vision and Image Understanding, 2004, 96(1) : 1-16. . 